\title{
Three-Dimensional Viscous Confinement and Cooling of Atoms by Resonance Radiation Pressure
}

\author{
Steven Chu, L. Hollberg, J. E. Bjorkholm, Alex Cable, and A. Ashkin \\ AT\&T Bell Laboratories, Holmdel, New Jersey 07733 \\ (Received 25 April 1985)
}

\begin{abstract}
We report the viscous confinement and cooling of neutral sodium atoms in three dimensions via the radiation pressure of counterpropagating laser beams. These atoms have a density of about $\sim 10^{6} \mathrm{~cm}^{-3}$ and a temperature of $\sim 240 \mu \mathrm{K}$ corresponding to a rms velocity of $\sim 60 \mathrm{~cm} / \mathrm{sec}$. This temperature is approximately the quantum limit for this atomic transition. The decay time for half the atoms to escape a $\sim 0.2-\mathrm{cm}^{3}$ confinement volume is $\sim 0.1 \mathrm{sec}$.
\end{abstract}

PACS numbers: $32.80 . \mathrm{Pj}$

The deflection of atoms by light resonant with an atomic transition was observed as early as 1933 by Frisch. ${ }^{1}$ Much later Ashkin ${ }^{2}$ pointed out that laser light can exert a substantial scattering force on an atom. He raised the possibility that this force could be used to trap atoms, and subsequently there have been numerous proposals to cool and ultimately trap neutral atoms. $^{3}$ Various experiments have been proposed that would utilize trapped atoms; these generally require long observation times and/or low atomic velocities. We report the demonstration of a confinement scheme based on the damping of atomic velocities. This scheme is not a trap, but can confine atoms in a small region in space for times on the order of $0.1 \mathrm{sec}$, and cool them to $\sim 240 \mu \mathrm{K}$, the quantum limit for our experimental conditions. For comparison, we note that two-dimensional radiative cooling has reduced the temperatures transverse to the motion of an atomic beam from 40 to $3.5 \mathrm{mK},{ }^{4}$ and atomic beams stopped by light have resulted in three-dimensional temperatures of $50-100 \mathrm{mK} .^{5}$ Laser cooling of electromagnetically trapped ions has resulted in ion temperatures between 5 and $100 \mathrm{mK}^{6}$

The basic physics of the viscous damping scheme is briefly outlined. Consider an atom irradiated by a laser beam tuned near a resonance line. For each photon absorbed, an atom receives a net change of momentum $\Delta p=h / \lambda$, where $\lambda$ is the wavelength. Since the subsequent reemission of the photon has no preferred direction, an average of many scattering events gives a net scattering force along the direction of the light. ${ }^{2}$ Hänsch and Schawlow ${ }^{7}$ noted that if counterpropagating beams were tuned to the low-frequency side of the absorption line, there would always be a net force opposing the velocity of an atom. For example, an atom moving with velocity $+v_{x}$ will blue shift into resonance with a laser beam propagating towards $-\hat{\mathbf{x}}$ and red shift out of resonance with the laser beam propagating towards $+\hat{\mathbf{x}}$. Thus, the atom is more likely to absorb photons going towards $-\hat{\mathbf{x}}$. With the use of six beams along $\pm \hat{\mathbf{x}}, \pm \hat{\mathbf{y}}$, and $\pm \hat{\mathbf{z}}$ and an averaging over many absorptions, the net effect is a viscous damping force $\mathbf{F}=-\alpha \mathbf{v}$ opposite the velocity of the atom. The cooling rate is $\mathbf{v} \cdot \mathbf{F}=-\alpha v^{2}$. The expression for the damping force which includes standing waves and saturation has been previously derived. ${ }^{8}$

In addition to the average force, statistical fluctuations must be considered. ${ }^{9}$ These fluctuations lead to heating. For a simple picture of the fluctuations, consider the momentum impulses on an atom due to the absorption and emission of photons. In the absence of damping, the atoms will execute a random walk in velocity, and although $\langle v\rangle=0,\left\langle v^{2}\right\rangle$ will increase linearly with the total number scattered photons. Increasing $\left\langle v^{2}\right\rangle$ corresponds to heating, as first observed by Bjorkholm et al. ${ }^{10}$ If we equate the heating and cooling terms, the steady-state kinetic temperature is obtained. In the absence of stimulated processes, the minimum kinetic energy for a two-level atom is given by $k T=\frac{1}{4} h \gamma$, where $\gamma$ is the width (FWHM) of the absorption line. If one includes stimulated processes, the minimum temperature is increased by a factor of 2. ${ }^{8}$ For sodium, $\gamma=10 \mathrm{MHz}$ and $T_{\min }=240 \mu \mathrm{K}$.

An estimate of the confinement time can be obtained by the observation that the motion of atoms in a viscous fluid of photons ("optical molasses") is analogous to diffusion in classical Brownian motion. The diffusion constant $D$ is given by the Einstein relation $D=k T / \alpha$, and for an infinite medium, $D=\left\langle x^{2}\right\rangle / 2 t$, where $\left\langle x^{2}\right\rangle$ is the mean square displacement after time $t$. However, an analysis based on an infinite medium overestimates the storage time. A more appropriate model is a viscous fluid surrounded by a spherical boundary (defined by the extent of the laser beams) such that the atoms that reach the boundary escape. If we assume an initial uniform concentration of atoms $n_{0}$, the average concentration $\bar{n}$ has been shown ${ }^{11}$ to vary as

$$
\bar{n}=n_{0} \frac{6}{\pi^{2}} \sum_{v=1}^{\infty} \frac{1}{v^{2}} e^{-D \pi^{2} v^{2} t / R^{2}} .
$$

The spherical-boundary modification of the randomwalk analysis reduces the storage time for our experimental conditions by a factor of 3.1 . 
The experimental apparatus is schematically shown in Fig. 1. We use a pulsed atomic beam source in order to simplify diagnostics. The beam of sodium atoms is produced by irradiation of a pellet of sodium metal with a $\sim 10$-nsec pulse from a frequencydoubled Nd-YAlG laser $(\sim 30-\mathrm{mJ}$ pulse focused to $\sim 5 \times 10^{-2} \mathrm{~cm}^{2}$ ). A hot plasma is formed which produces sodium ions, suprathermal atoms with average energy of $\sim 3.5 \mathrm{eV}$, and a small fraction of thermal atoms at $\sim 1000 \mathrm{~K}$. The source of atoms is apertured to produce a directed $0.6-\mathrm{cm}$-diam atomic beam at the confinement region shown in Fig. 1.

Since our "optical molasses" can only capture atoms moving with velocities $\leq 3 \times 10^{3} \mathrm{~cm} / \mathrm{sec}$, we slow some of the atoms in the atomic beam by using a counterpropagating laser beam. Following Ertmer et al. ${ }^{5}$ we use an electro-optic modulator to generate a frequency-shifted sideband which can be swept in frequency to stay in resonance with the changing Doppler shift of the atomic resonance frequency as the atoms slow down. The laser intensity used to slow (precool) the atoms is $-120 \mathrm{~mW} / \mathrm{cm}^{2}$. Atoms with initial velocities of $\sim 2 \times 10^{4} \mathrm{~cm} / \mathrm{sec}$ and less are slowed down in less than $5 \mathrm{~cm}$ to velocities $\sim 2 \times 10^{3} \mathrm{~cm} / \mathrm{sec}$. After slowing for $0.5 \mathrm{msec}$, the precooling beam is shut off by an acoustic-optic modulator, and the atoms then drift into the region defined by the six intersecting laser beams where they are cooled and viscously confined. The precooling laser beam and the confining beams are obtained from the combined beams of two cw actively stabilized, ring dye lasers. The lasers are operated at frequencies differing by $\sim 1.7 \mathrm{GHz}$ to prevent optical pumping of the sodium ground state.
The power of each confining beam is between 4 and 20 $\mathrm{mW}$, and the beam radius is $w_{0}=0.36 \mathrm{~cm}$. Despite the fact that the vast majority of atoms are not slowed down, the pulsed beam is sufficiently intense that confinement densities on the order of $10^{6}$ atoms $/ \mathrm{cm}^{3}$ are obtained. At these densities, the cloud of confined atoms is clearly visible by eye.

An averaged fluorescence signal (sixteen pulses) as a function of time is shown in Fig. 2. Pulse-to-pulse amplitude fluctuations are $-30 \%$. The initial abrupt spike is due to fast atoms passing rapidly through the interaction region. The baseline is the scattered light level obtained by blockage of the precooling beam. If any of the confining dimensions is blocked, a fluorescence pulse $\leqslant 1 \mathrm{msec}$ long is seen as a result of the passage of the slowed atoms through the interaction region. In addition, the laser frequencies must be tuned to the low-frequency side of the absorption lines and be critically tuned with respect to each other. In Fig. 2, we plot the number of atoms remaining in the observation region as a function of $D t / R^{2}$, where $D$ is the diffusion constant, $t$ is the time, and $R$ is an effective radius to the spherical boundary. We extract a value for $D$ by scaling the horizontal axis of the experimental data to match to the theoretical decay given by Eq. (1). If we take $R=0.4 \mathrm{~cm}$ (where the intensity of the laser beam is $\simeq 0.1$ of the peak intensity), the decay curves give us an effective diffusion constant, $D_{\text {eff. }}$. Using a computed value of $\alpha=\alpha_{\max }=5.8 \times 10^{-18}$ $\mathrm{g} / \mathrm{sec},{ }^{8}$ we obtain an upper limit on the temperature of $T_{\max }=D_{\text {eff }} \alpha_{\max } / k=1.9 \mathrm{mK}$. The actual temperature is expected to be lower since we have ignored drift velocities due to beam intensity imbalance, intensity

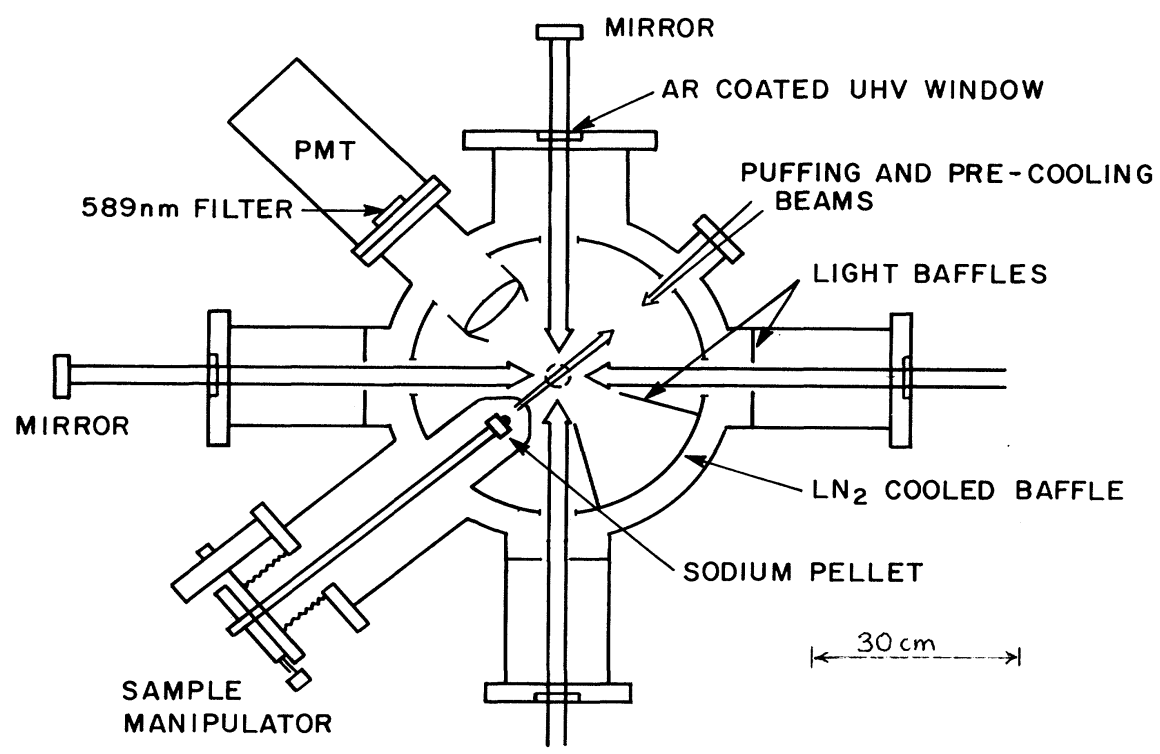

FIG. 1. Schematic of the vacuum chamber and intersecting laser beams and atomic beam. The vertical confining beam is indicated by the dashed circle. The "puffing" beam is from the pulsed YAlG laser. 


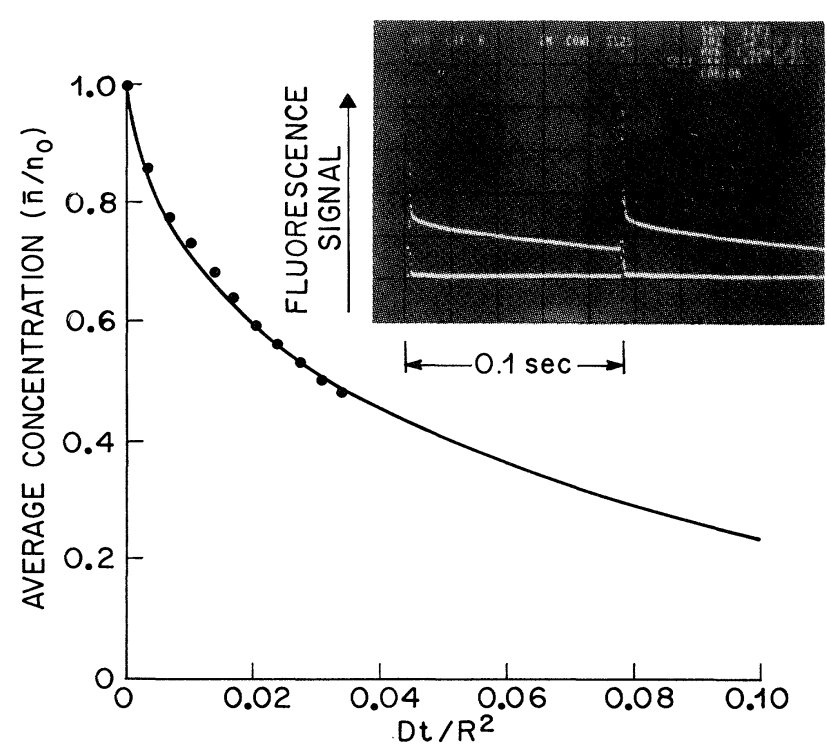

FIG. 2. Fluorescence signal as a function of time is shown in the inset. The baseline shows the scattered light level. The confinement region is loaded every $0.1 \mathrm{sec}$, governed by the repetition rate of the YAlG laser. The fraction of atoms remaining in the observation region is plotted as a function of $D t / R^{2}$. The solid line is the theoretical calculation based on the modified random walk.

hot spots, and beam misalignment, all of which increase $D_{\text {eff }}$ but do not substantially increase the temperature of the atoms. For example, if the counterpropagating beams are imbalanced by $2 \%$, the drift velocity is $v_{\text {drift }}=3 \mathrm{~cm} / \mathrm{sec}$, and the storage time will be $R / v_{\text {drift }} \sim 0.13 \mathrm{sec}$.

A direct measurement of the temperature of the cooled atoms is obtained by a time-of-flight technique. After a 15-msec cooling and confinement time, all six beams are turned off in $-0.1 \mathrm{msec}$ and left off for a variable time. During that time, the atoms will leave the observation region ballistically with their instantaneous velocities. We show in the inset of Fig. 3 an example time-of-flight measurement for a $9.1-\mathrm{msec}$ light-off time. Given an initial uniform spherical distribution of atoms with a Maxwell-Boltzmann distribution of velocities, we calculate the fraction of atoms remaining in the observation region as a function of the light-off time. To fit the data with calculated decay curve, we introduce a $1.4-\mathrm{msec}$ time shift in the theoretical curve to account for the fact that the actual atomic distribution will be depleted near the edge of the sphere by Brownian diffusion to the escape boundary. ${ }^{11}$ The measured fraction of atoms remaining and the theoretical curve are plotted in Fig. 3. Additional uncertainty in the temperature measurement arises from the fact that our Gaussian-type beam profiles must be convoluted with saturation effects and the acceptance volume of our phototube. Note that the tem-

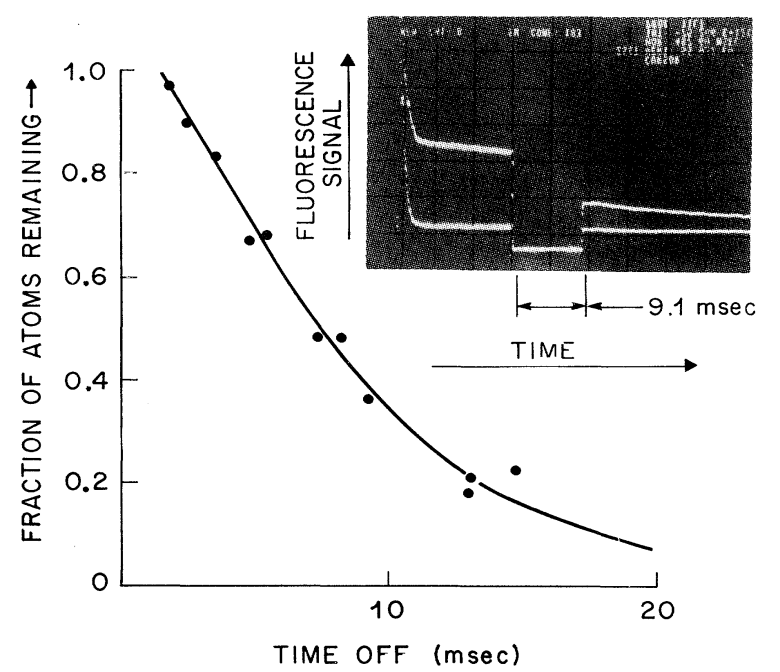

FIG. 3. Inset shows the effect of blocking the confining laser beams $15 \mathrm{msec}$ after the YAlG laser fires. The horizontal trace is the scattered light level. The fraction of atoms remaining is plotted as a function of the time-off period. The solid line is the theoretical curve.

perature depends on $R_{\text {eff }}^{2}$, where $R_{\text {eff }} \cdot$ is an effective observation radius. Using a fairly conservative value of $R_{\text {eff }}=0.4 \mathrm{~cm}$, we obtain $T=240 \pm 200 \mu \mathrm{K}$.

In summary, we have confined sodium atoms in a $\sim 0.2-\mathrm{cm}^{3}$ volume for times on the order of $0.1 \mathrm{sec}$ and cooled them to temperatures approaching the quantum limit. These kinetic temperatures are significantly lower than the electromagnetically trapped ions ${ }^{6}$ or stopped atoms ${ }^{5}$ previously reported. The low velocities and long confinement times that we have achieved can increase the observation and/or coherence time for atoms, opening up new possibilities in areas such as collisions, quantum statistics, and precision spectroscopy. In addition, the low temperatures make possible the efficient loading of atom traps. Such traps will help in reaching higher densities and possibly temperatures as low as $10^{-6} \mathrm{~K}^{3}$ At these temperatures, the de Broglie wavelength of the atoms is on the order of $1 \mu \mathrm{m}$. Even modest densities of these ultracold atoms (which can be either fermions or bosons) should reveal interesting physics.

We acknowledge helpful discussions with J. P. Gordon and assistance from $\mathrm{L}$. Buhl in the preparation of the $\mathrm{LiTaO}_{3}$ crystal used in the electro-optic modulator.

1O. R. Frisch, Z. Phys. 86, 42 (1933).

${ }^{2}$ A. Ashkin, Phys. Rev. Lett. 24, 156 (1970), and 25, 1321 (1970).

${ }^{3}$ See, for example, A. Ashkin, Science 210, 1081 (1980), and Prog. Quantum Electron. 8, 204 (1984); for recent proposals, see D. E. Pritchard, Phys. Rev. Lett. 51, 1336 
(1983); A. Ashkin, Opt. Lett. 9, 454 (1984); J. Dalibard, S. Reynaud, and C. Cohen-Tannoudji, J. Phys. B 17, 4577 (1984).

${ }^{4}$ V. I. Balykin, V. S. Letokhov, and A. I. Sidorov, Pis'ma Zh. Eksp. Teor. Fiz. 40, 251 (1984) [JETP Lett. 40, 1026 (1984)].

5J. Prodan, A. Migdall, W. D. Phillips, I. So, H. Metcalf, and J. Dalibard, Phys. Rev. Lett. 54, 992 (1985); W. Ertmer, R. Blatt, J. L. Hall, and M. Zhu, Phys. Rev. Lett. 54, 996 (1985).

6W. Neuhauser, M. Hohenstatt, P. E. Toschek, and H. Dehmelt, Phys. Rev. A 22, 1137 (1980); D. J. Wineland and W. M. Itano, Phys. Lett. 82A, 75 (1981); W. Nagourney, G. Janik, and H. Dehmelt, Proc. Natl. Acad. Sci. U.S.A. 80, 643 (1983).
${ }^{7}$ T. W. Hänsch and A. L. Schawlow, Opt. Commun. 13, 68 (1975).

8J. P. Gordon and A. Ashkin, Phys. Rev. A 21, 1606 (1980); R. J. Cook, Phys. Rev. A 20, 224 (1979), and Phys. Rev. Lett. 44, 976 (1980).

${ }^{9}$ A. Yu. Pusep, Zh. Eksp. Teor. Fiz. 70, 851 (1976) [Sov. Phys. JETP 43, 441 (1976)]; V. S. Letokhov, V. G. Minogin, and B. D. Pavlik, Zh. Eksp. Teor. Fiz. 72, 1328 (1977) [Sov. Phys. JETP 45, 698 (1977)]; J. L. Picqué, Phys. Rev. A 19, 1622 (1979); D. J. Wineland and W. M. Itano, Phys. Rev. A 25, 35 (1982).

10J. E. Bjorkholm, R. R. Freeman, A. Ashkin, and D. B. Pearson, Opt. Lett. 5, 111 (1980).

${ }^{11}$ N. A. Fuchs, The Mechanics of Aerosols (Pergamon, Oxford, 1964), pp. 193-200. 


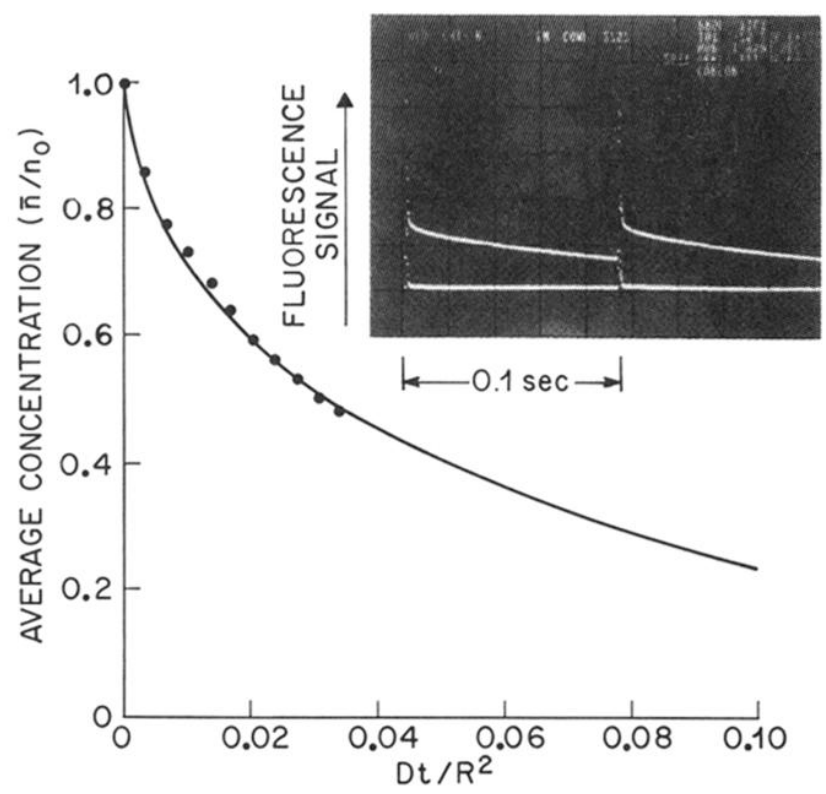

FIG. 2. Fluorescence signal as a function of time is shown in the inset. The baseline shows the scattered light level. The confinement region is loaded every $0.1 \mathrm{sec}$, governed by the repetition rate of the YAlG laser. The fraction of atoms remaining in the observation region is plotted as a function of $\mathrm{Dt} / R^{2}$. The solid line is the theoretical calculation based on the modified random walk. 


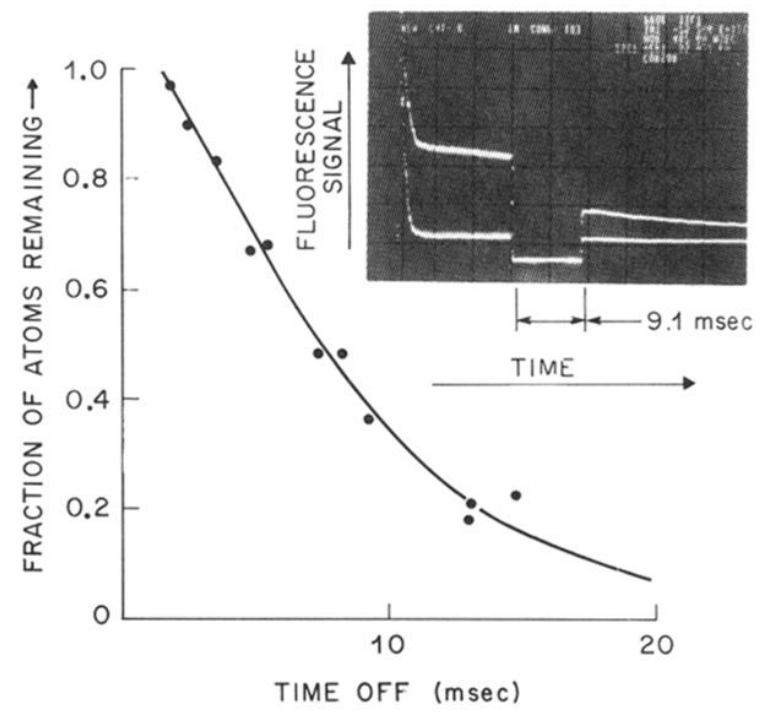

FIG. 3. Inset shows the effect of blocking the confining laser beams $15 \mathrm{msec}$ after the YAlG laser fires. The horizontal trace is the scattered light level. The fraction of atoms remaining is plotted as a function of the time-off period. The solid line is the theoretical curve. 